\title{
Genetic Algorithm-based Fuzzy Optimization Neural Network Model for WSNs Performance Evaluation
}

\author{
Fawang Han \\ Nanjing Forest Police College, Jiangsu Nanjing 210023, China \\ hanfawang@163.com
}

\begin{abstract}
In recent years, wireless sensor network is becoming the hottest research fields, which opens up a wealth of opportunities for many application domains. Comprehensive performance of WSNs is important for planning and management of a construction network. In order to deal with the deficiency of existing evaluation methods for WSNs performance, an intelligent evaluation model based on fuzzy optimization neural network model is proposed, which introduces genetic algorithm to optimize the connection weight of the neural network model to achieve approximate optimal solution. The weights are to be regarded as initial values for the next step that a neural network is tuned finely further. Fuzzy optimization model is as activation function of the neural network, therefore the model has explicit physical meanings. This model is applied to evaluate WSNs performance of some samples, and the comparative analysis with other models shows that the model improves evaluation precision and efficiency and is practical. The first part is the research status and related problems. The second part is the establishment of evaluation model. The last part is the experimental analysis and conclusion.
\end{abstract}

Keywords: WSNs, fuzzy optimization, neural network model, Genetic algorithm

\section{Introduction}

In recent years, wireless sensor network is becoming the hottest research fields concerning in the international community, involving cross multidisciplinary and highly integrated knowledge [1-2]. The wireless sensor network has very vast application prospect and potential utility value, opens up a wealth of opportunities for military management, industry and agriculture, city management, Bio Medical, environmental monitoring, emergency rescue and disaster relief, anti-terrorism, remote control of hazardous area and many other important areas [3-6]. There have been several large-scale sensor network deployments reported during the past years, including Vigil-Net for field surveillance [7], Mote lab that provides an indoor test bed [8], Sensor Scope for weather monitoring in the wild [9], and Trio which enables a large-scale solar-powered sensor network [10]. WSNs has been highly paid more attention by academic and industrial circles in many countries, and was regarded as one of the most shining technology which has the huge influence on twenty-first century.

The current research on WSNs mainly concentrated in the protocol stack development, network topology design, algorithms in each protocol layer, application prospects and other aspects[11-13]. There are few articles about performance rank in WSNs, we need a comprehensive guidance theory to evaluate the network performance of WSNs, which can be agreed by most researchers [14-15]. A number of empirical studies present network measurement results in many aspects, with emphasis on understanding the complex and nonideal behavior of low power wireless communications[16-18]. In current researches, 
according to the performance index of wireless sensor network, there is no uniform standard. There is some related research for ordinary IP nets, and two major international institutions research and analysis, set up the parameters of IP network performance, which are speed, accuracy, dependability, availability and so on[22-23]. On the one hand, WSNs performance indexes usually are separately considered [24-25], which neglects the crossover impact; on the other hand, weight is ignored [26]. At present, traditional network performance comprehensive evaluation models are regression analysis model, system analysis model, and gray theory model and so on [27-29]. However, the regression analysis model is insufficient to accurately characterize the complex connection between WSNs performance measured and expert evaluation results, human factors in the system analysis method is too heavy, grey theory model is too dependent on the evaluation experience range. Some aspects of the method in the prediction accuracy and efficiency have achieved gradual improvement, but how to make the physical meaning of the neural network model more clear, and easy to understand.

Therefore, with considering the intricate characters of WSNs in different applications, we integrate genetic algorithm and fuzzy optimization neural network (FONN) to build a full advantage WSNs performance evaluation model. In this paper, firstly, we build fuzzy optimization neural network model, which contains fuzzy optimization and neural network. Secondly, an improved fuzzy optimization neural network model based on the genetic algorithm is suggested to study the WSNs performance. Finally, we apply Open network simulation software to implement simulation experiments; the evaluation results show us that the proposed model is efficient and effective.

\section{Fuzzy Optimization Neural Network Model}

\subsection{Fuzzy Optimization Model}

Superiority and inferiority are two antithesis concepts, which have both difference and collective dimension in the two extreme points with intermediary transition. Therefore, it is called fuzzy optimization. Fuzzy optimization is carried out in the finite domain of nondominated solution set. To a certain standard, this is relatively preferred. We assume that the system has a scheme set composing of $n$ plans to meet the constraints, with $m$ targets (evaluation index) on schemes for evaluation. The eigenvalue matrix is as following:

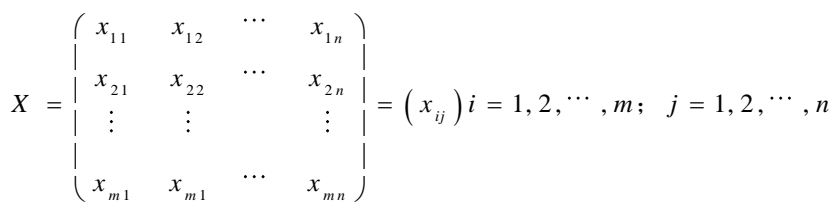

In which $x_{i j}$ is the eigenvalue of $i$ th index in $j$ th WSNs. There are two kinds of target eigenvalue, one type in which the bigger of the value the better, the other type in which the smaller of the value the better. The formulation of the former can be represented as follows:

$$
r_{i j}=\left\{\begin{array}{l}
1 ; \quad x_{i \max } \\
x_{i j} / x_{i \max } ; \\
0 ; \quad 0
\end{array}\right.
$$

And the formulation of the latter can be represented as follows: 


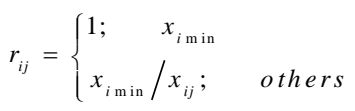

Then we can transfer the eigenvalue matrix $X$ into $R$

$$
R=\left\{\begin{array}{cccc}
r_{11} & r_{12} & \cdots & r_{1 n} \\
r_{21} & r_{22} & \cdots & r_{2 n} \\
\vdots & \vdots & & \vdots \\
r_{m 1} & r_{m 1} & \cdots & r_{m n}
\end{array}\right)=\left(r_{i j}\right)
$$

According to the relative membership degree defined, inferior and superior are respectively in two little reference pole, and the objective vector of the inferior and superior schemes are respectively:

$$
\begin{aligned}
& b=\left(\begin{array}{llll}
0 & 0 & \cdots & 0
\end{array}\right) \\
& g=\left(\begin{array}{llll}
1 & 1 & \cdots & 1
\end{array}\right)
\end{aligned}
$$

The weight matrix of $i$ th index in $j$ th WSNs is as following:

$$
W=\left\{\begin{array}{cccc}
w_{11} & w_{12} & \cdots & w_{1 n} \\
w_{21} & r_{22} & \cdots & w_{2 n} \\
\vdots & \vdots & & \vdots \\
w_{m 1} & w_{m 1} & \cdots & w_{m n}
\end{array}\right)=\left(w_{i j}\right)
$$

In which $w_{i j}$ is the weight of $i$ th index in $j$ th plan, given a random WSNs, $\sum_{i=1}^{m} w_{i j}=1$. However, the distance between $j$ th WSNs and the superior or inferior schemes is:

$$
\begin{aligned}
& d_{j g}=\sum_{i=1}^{m} w_{i j}\left(1-r_{i j}\right)=1-\sum_{i=1}^{m} w_{i j} r_{i j} \\
& d_{j b}=\sum_{i=1}^{m} w_{i j}\left(r_{i j}-0\right)=\sum_{i=1}^{m} w_{i j} r_{i j}
\end{aligned}
$$

Assume that relative membership degree of $j$ th WSNs comparing to the superior and inferior schemes is $u_{j}$ and $u_{j}^{c}$, and $u_{j}^{c}=1-u_{j}$. Defining membership degree as weight, we get the weight distance:

$$
D_{j g}=u_{j} d_{j g}, D_{j b}=u_{j}^{c} d_{j b}=\left(1-u_{j}\right) d_{j b}
$$

In order to solve the optimal value of relative membership degree of $j$ th WSNs, minimize the square sum of weight distance away from the superior and inferior schemes, and the objective function is:

$$
\min F\left(u_{j}\right)=D_{j b}^{2}+D_{j g}^{2}=u_{j}^{2}\left(1-\sum_{i=1}^{m} w_{i j} r_{i j}\right)^{2}+\left(1-u_{j}\right)^{2}\left(\sum_{i=1}^{m} w_{i j} r_{i j}\right)^{2}
$$

If $d F\left(u_{j}\right) / u_{j}=0$, we obtain the fuzzy optimization model: 


$$
u_{j}=\frac{1}{\left\lfloor+\left\lfloor\frac{1-\sum_{i=1}^{m} w_{i j} r_{i j}}{\left\lfloor\sum_{i=1}^{m} w_{i j} r_{i j}\right.}\right\rfloor\right.}=\frac{1}{1+\left(\frac{d_{j g}}{d_{j b}}\right)^{2}} \frac{1}{1+\left(\frac{1-d_{j b}}{d_{j b}}\right)^{2}}
$$

The physical meaning is that the relative membership degree of jth WSNs $u_{j}>0.5$, if $d_{j g}>d_{j b}$; if $d_{j g}<d_{j b}, u_{j}=0.5$; and if $d_{j s}=0$, the jth WSNs is the best scheme, $u_{j}=1$; if $d_{j s}=1$, then $u_{j}=0$. Therefore, we can use fuzzy optimization model to describe the incentive function in neuron network system, make the incentive function of node neurons have distinct physical meaning, and optimization is the essence of the human brain.

\subsection{Neural Network Model}

In a given neuron network, the number of nodes in input layer nodes is $m$, hidden layer node is $l$, output layer nodes is $g$. To facilitate the representation, we take $i$ as input layer node, $k$ as hidden layer node, $p$ as output layer node in Figure 1.

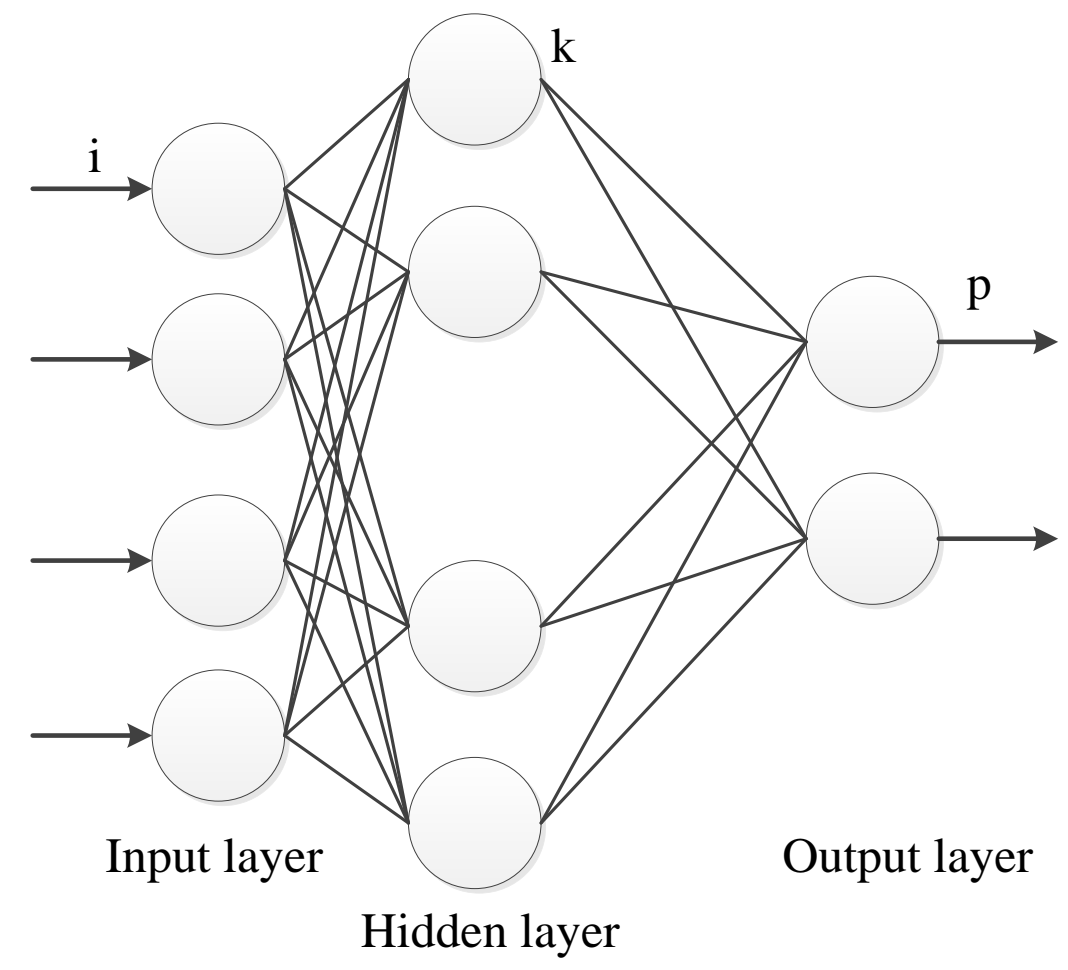

Figure 1. Neural Network Framework

There are m samples, the input value of ith sample is $r_{i j}, i=1,2, \cdots, m j=1,2, \cdots, n$. Node $i$ in input layer deliveries information to node $k$ in hidden layer directly, and input value is equal to output value, $u_{i j}=r_{i j}$.

According to node $k$ in hidden layer, the input value is $I_{k j}=\sum_{i=1}^{m} w_{i k} r_{i j}$, and its output value is: 


$$
u_{k j}=\frac{1}{1+\left\lfloor\left(\sum_{i=1}^{m} w_{i k} r_{i j}\right)^{-1}-1\right\rceil^{2}}=\frac{1}{1+\left(I_{k j}^{-1}-1\right)^{2}}
$$

In the formula, $w_{i k}$ is connection weight between node $i$ and $k$.

According to node $p$ in output layer, the input value is ${ }_{p j}=\sum_{k=1}^{m} w_{k p} r_{k j}$. And its output value is:

$$
u_{p j}=\frac{1}{1+\left\lceil\left(\sum_{k=1}^{m} w_{k p} r_{k j}\right)^{-1}-1\right\rceil^{2}}=\frac{1}{1+\left(I_{p j}^{-1}-1\right)^{2}}
$$

In the formula, $w_{i k}$ is connection weight between hidden layer and output layer.

The actual output value $u_{p j}$ is responsive to input $r_{i j}$ in fuzzy optimal selection of neural network. Assuming that the expected output value of the jth sample is $M\left(u_{p j}\right)$, we can calculate the square error as follows:

$$
E=\frac{1}{n} \sum_{j=1}^{n} E_{j}=\frac{1}{2 n} \sum_{j=1}^{n} \sum_{p=1}^{g}\left(u_{p j}-M\left(u_{p j}\right)\right)^{2}
$$

Then, the connection weight between nodes of the hidden layer and the output layer node is adjustment:

$$
\Delta w_{k p}=\frac{2 \eta}{n} \sum_{j=1}^{n} u_{p j}^{2} u_{k j}\left|\frac{\left\lceil 1-\sum_{k=1}^{m} w_{k p} u_{k j}\right.}{\left\lfloor\left(\sum_{k=1}^{m} w_{k p} u_{k j}\right)^{3}\right.}\right|^{g} \times \sum_{p=1}^{g}\left(M\left(u_{p j}\right)-u_{p j}\right)
$$

In the formula, $\eta$ is learning coefficient. The connection weight between the input layer nodes and the hidden layer nodes is also an adjustment:

$$
\Delta w_{i k}=\frac{2 \eta}{n} \sum_{j=1}^{n} r_{i j} w_{k p} u_{k j}^{2}\left|\frac{\left|1-\sum_{k=1}^{m} w_{k p} u_{k j}\right|}{\left.\mid \sum_{k=1}^{m} w_{i k} r_{i j}\right)^{3}}\right|^{\top} \delta_{p j}
$$

The weight adjustment formula is as follow:

$$
\begin{gathered}
w_{i k}(t+1)=w_{i k}(t)+\Delta w_{i k}(t+1)+\alpha \Delta w_{i k}(t) \\
w_{k p}(t+1)=w_{k p}(t)+\Delta w_{k p}(t+1)+\alpha \Delta w_{k p}(t)
\end{gathered}
$$

In which $\alpha$ is momentum coefficient, $0<\alpha<1$.

Using the model referred to above, we can determine the network connection weightts, according to the training algorithm of neural network, and make the error between the actual output and the expected output error minimized.

\section{Improved Fuzzy Optimization Neural Network Model based on Genetic Algorithm}

The neural network model is easy to appear provincial minimum, and has the disadvantage of slow convergence rate. Therefore we integrate genetic algorithm which gets the 
characteristic of global search and efficiency, with fuzzy optimization neural network algorithm together.

\subsection{To Determine the Encoding Method}

Floating point encoding method has lots of advantages, such as larger range of representation, high precision, large search space, and is easy to mix with other methods. Due to computational precision, efficiency and convenience, we build our model using floating point decoding method, which resolves our problem of encoding selection.

\subsection{The Population Initialization}

In the premise of guaranteeing the neural network sensitivity, the net input of each node is near to zero. In order to create more feasible solutions when we optimize FONN weight values using GA, we set the range of the initial population weights [2, -2].

\subsection{Fitness calculation and set other parameters of GA}

GA fitness is very good characterization of individual chromosomes, the larger the fitness is, the better the performance of chromosomes. We apply $f(E)=1 /(1+10 \times E)$ as fitness function in the model, after FONN training 25times and the output error is E which is the average relative error between the actual output and the expert scoring. Setting population size at 35, terminating conditions are $E \leq \varepsilon_{1}$ or to obtain the maximum number of iterations as 15. We employ selection operation by the roulette wheel method and crossover operation in uniform arithmetic crossover. The crossover probability is 0.6 , and the mutation probability is 0.05 .

\subsection{The process of evaluation model}

In this paper, we apply the Delphi program to realize the evaluation process, the concrete steps as showed in Figure 2.

(1)Firstly, determine the encoding method, terminating conditions, fitness function, other parameters of GA, initialization population size;

(2)Decoding to the solution space, after training 25 times, the output error is $E$, then we calculate the individual chromosome fitness $f(E)=1 /(1+10 \times E)$;

(3)Judge whether to meet the termination conditions of genetic algorithm ( $E \leq \varepsilon_{1}$ or to obtain the maximum number of iterations as 15 ), if to meet, then turn into (5), otherwise we continue step by step;

(4) Genetic operation such as selection operation, crossover operation and mutation operation, generate new population and turn to(2);

(5) Using BP network, we make slight adjustment of the approximate optimal solutions which is obtained by GA search, improve the accuracy of solutions until it reaches termination conditions, and then output results. 


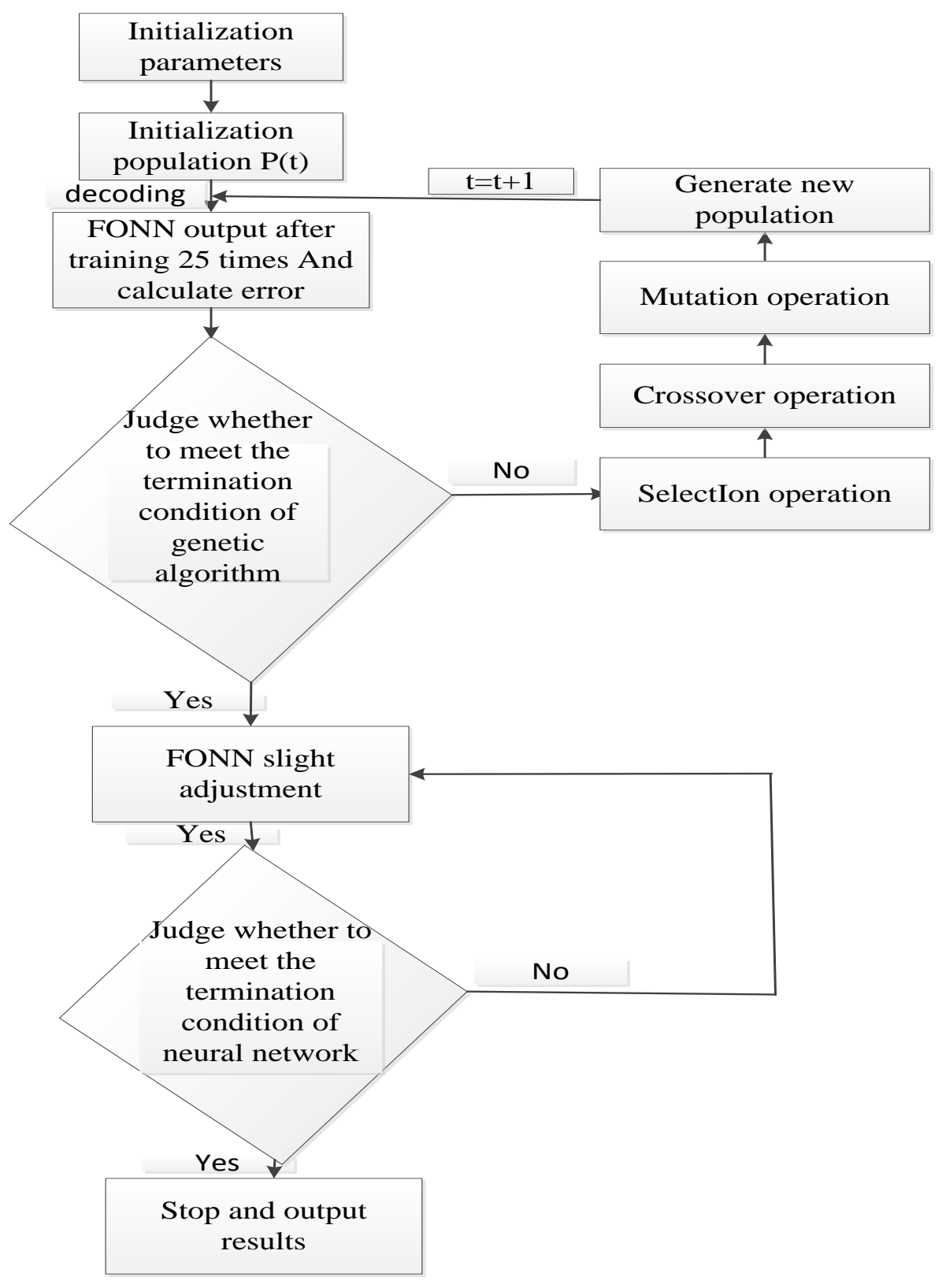

Figure 2. The Process of WSNs Performance Evaluation Model

\section{A Numerical Example}

To demonstrate the use of the singular metrics for wireless sensor network reliability and to explore the relationships between these metrics, a series of tests were conducted with a single radio transmitting to a receiver. Since many applications will require low data-rate communications, hardware was selected that conformed to the IEEE 802.15.4 standard for low-rate wireless personal area networks $(2.4 \mathrm{GHz}$ bands). In this paper, the performance evaluation of wireless sensor network is based on ZigBee technology, using network simulation software OPNET to build separate networks. Based on the simulated measurement, we collect network performance data, and evaluate the comprehensive performance of WSNs. 


\subsection{Select the Input and Output Parameters}

Network performance reflects the quality of the network to provide communications services, is the embodiment of the characteristics of the network itself, can be composed of a series of index definitions and description. The three most commonly used indicators of network performance in networks are bandwidth, delay and packet loss rate. We select time delay, packet loss rate, and throughput as the model input parameters, and expert scoring as model output parameter.

\subsection{Input and Output Data Preprocessing}

We utilize the Sigmoid distribution transformation method to normalization process the input data in improved fuzzy optimization neural network model. In this paper, the Sigmoid distribution transformation forma is:

$$
y_{i}=\frac{1}{1+b e^{-a x_{i}}}
$$

In the formula $x_{i}$ is the initial value of evaluation index, $y_{i}$ is the value of evaluation index after distribution transform, $a, b$ is undetermined parameters, as show in Table 1.

Table 1. Parameters for Transformation WSNs Performance Evaluation Indices

\begin{tabular}{|c|c|c|c|c|c|c|}
\hline \multirow{2}{*}{ Index } & \multicolumn{2}{|c|}{ The original index optimal } & \multicolumn{2}{c|}{ The original index inferior } & \multicolumn{2}{c|}{$\begin{array}{c}\text { undetermined } \\
\text { parameters }\end{array}$} \\
\cline { 2 - 7 } & $x_{i}$ & $y_{i}$ & $x_{i}$ & $y_{i}$ & $\mathrm{a}$ & $\mathrm{b}$ \\
\hline time delay & 0.007453 & 0.75 & 0.092256 & 0.4 & -0.156 & 0.054 \\
\hline $\begin{array}{c}\text { packet } \\
\text { loss rate }\end{array}$ & 0.002754 & 0.75 & 0.066176 & 0.4 & 1.254 & 0.653 \\
\hline throughput & 43753.45 & 0.75 & 24638.91 & 0.4 & 0.842 & 10.245 \\
\hline
\end{tabular}

\subsection{Analysis of the results}

The generalization ability of the model is a very important aspect of our scientific evaluation model. For testing the generalization ability, we build the rank model using the 25 data sets collected by simulation, and divide it into 5 groups respectively, 4 groups as the training samples and the rest as testing samples(as shown in Table 2); In order to verify the stability, we carry out 5 times of consecutive training for each model; To test the effectiveness of the evaluation model, we compare results with other methods, such as regression analysis, BP neural network model and genetic neural network models.

Table 2. Statistical Table of Subjective Scores and Practica1data of WSNs

\begin{tabular}{|c|c|c|c|c|}
\hline $\begin{array}{c}\text { Number of } \\
\text { WSNs }\end{array}$ & scores & time delay & packet loss rate & throughput \\
\hline 1 & 3.12 & 0.010150 & 0.013690 & 38095.49 \\
\hline 2 & 4.25 & 0.070866 & 0.002560 & 29374.13 \\
\hline 3 & 5.36 & 0.092256 & 0.001167 & 28449.55 \\
\hline 4 & 5.02 & 0.011366 & 0.066176 & 43753.45 \\
\hline
\end{tabular}




\begin{tabular}{|l|l|l|l|l|}
\hline 5 & 4.32 & 0.007456 & 0.002754 & 28027.18 \\
\hline 6 & 3.62 & 0.008310 & 0.002895 & 28027.18 \\
\hline 7 & 4.01 & 0.082839 & 0.002895 & 46038.35 \\
\hline 8 & 2.98 & 0.008356 & 0.012486 & 40385.54 \\
\hline 9 & 4.23 & 0.010455 & 0.003874 & 35548.68 \\
\hline 10 & 5.16 & 0.007234 & 0.042158 & 35678.47 \\
\hline
\end{tabular}

The training results of evaluation model is shown in Table 3 , and terminating conditions of GA is $E \leq 0.04$, terminating conditions of neural network is $E \leq 0.02, C_{1}, C_{2}, C_{3}$ are correlation coefficient between the data of training samples, testing samples, expert scores and regression predict data. We can see clearly that the correlation coefficient achieve 0.95 above, absolute error basically is under 0.1 . With the continuous training, the model often converges to the same value point.

Table 3. Training Results of Evaluation Model

\begin{tabular}{|c|c|c|c|c|c|}
\hline Training sequence & $\begin{array}{c}\text { Training } \\
\text { times }\end{array}$ & Error & $C_{1}$ & $C_{2}$ & $C_{3}$ \\
\hline 1 & 1954 & 0.06 & 0.972 & 0.941 & 0.968 \\
\hline 2 & 1854 & 0.07 & 0.969 & 0.936 & 0.962 \\
\hline 3 & 2000 & 0.08 & 0.975 & 0.946 & 0.958 \\
\hline 4 & 2000 & 0.06 & 0.980 & 0.953 & 0.972 \\
\hline 5 & 1689 & 0.05 & 0.973 & 0.924 & 0.963 \\
\hline
\end{tabular}

As showed in Table 4, compared with GANN model, population size of is 50, iteration for more than 1200 generations to obtain ideal solution; and the population size of our model is 40 , only 15 iterations to get the ideal solution.

Table 4. Comparison Between Evaluation Model and GANN Model

\begin{tabular}{|c|c|c|}
\hline Model & Our model & GANN \\
\hline Population size & 40 & 50 \\
\hline Number of iterations & 15 & 1205 \\
\hline Times of slight adjustment & $<200$ & No \\
\hline Correlation coefficient & $>0.95$ & $>0.92$ \\
\hline
\end{tabular}

Fuzzy optimization model is as activation function of a neural network, therefore the model has explicit physical meanings. This model is applied to evaluate WSNs performance of some samples, and the comparative analysis with other models shows that the model improves evaluation precision and efficiency and is practical.

\section{Conclusion}

In recent years, wireless sensor network is the hottest research fields concerning in the international community, involving cross multidisciplinary and highly integrated knowledge. It integrates sensor technology, embedded computing technology, modern network, wireless communication technology and distributed information processing technology. The current 
research on WSNs mainly concentrated in the protocol stack development, network topology design, algorithms in each protocol layer, application prospects and other aspects. There are few articles about performance rank in WSNs. We need a comprehensive guidance theory to evaluate network performance of WSNs. In this paper, firstly, we build fuzzy optimization neural network model, which contains fuzzy optimization and the neural network. Secondly, an improved fuzzy optimization neural network model based on genetic algorithm is suggested to study the WSNs performance. Finally, we apply Open network simulation software to implement simulation experiments; the evaluation results show us that the proposed model is efficient and effective.

\section{Acknowledgements}

This paper is supported by the year in 2014, Nanjing Forest Police College, "The Fundamental Research Funds for the Central Universities". The project number is LGYB201412.

\section{References}

[1] Y. Sanakarasubfamaniam, "Wireless sensor networks: A survey, Computer Network", vol. 38, no. 4, (2002), pp. 93-422

[2] M. Kintner-Meyer, M. R. Brambley, T. A. Carlon and N. N. Bauman, "Wireless sensors: technology and cost-savings for commercial buildings, Teaming for Efficiency: Proceedings of the 2002 ACEEE Summer Study on Energy Efficiency in Buildings", (2002), pp. 121-134.

[3] W. M. Healy, "Lessons learned in wireless monitoring", ASHRAE Journal, vol. 47, no. 10, (2005), pp. 54-58.

[4] J. Raimo, "Wireless mesh controller networks", ASHRAE Journal, vol. 48, no. 10, (2006), pp. 34-38.

[5] D. A. Swyt, "An Assessment of the United States Measurement System: Addressing Measurement Barriers to Accelerate Innovation", NIST Special Publication1048, (2007).

[6] W. M. Healy and W. S. Jang, "Practical Challenges in Wireless Sensor Network Use in Buildings", NIST Technical Note 1604, (2008).

[7] IEEE 802.15.4 Wireless MAC and PHY Specifications for Low Rate Wireless Personal Area Networks (WPANs), Piscataway, NJ, IEEE, (2006).

[8] X. Boyen, "Multipurpose identity-based key, a Swiss army knife foridentity-based cryptography In: Proceedings of the 23rd international conference on advances in cryptology", Lecture notes in computer science, vol. 2729, (2003), pp. 383-399.

[9] C. Huang and D. Du, "New constructions on broadcast encryption and key pre-distribution schemes In: Proceedings of IEEE INFOCOM05", Miami: IEEE Press, (2005), pp. 515-23.

[10] D. Ioannis, A. Ribeiro and B. Georgios, "Consensus in ad hoc WSNs with noisy links-Part I: Distributed estimation of deterministic signals”, Signal Processing, IEEE Transactions on 56.1 (2008), pp. 350-364.

[11] A. Mpitziopoulos, et al., "A survey on jamming attacks and countermeasures in WSNs", Communications Surveys \& Tutorials, IEEE, vol. 11, no. 4, (2009), pp. 42-56.

[12] Stavrou, Eliana, and Pitsillides, "A survey on secure multipath routing protocols in WSNs", Computer Networks, vol. 54, no. 13, (2010), pp. 2215-2238.

[13] P. Wright and D. Dornfeld and N. Ota, "Condition monitoring in end-milling using wireless sensor networks (WSNs)", Transactions of NAMRI/SME, vol. 36, (2008), pp. 177-183.

[14] D. Jing, R. Han and S. Mishra, "A performance evaluation of intrusion tolerant routing in wireless sensor networks: Information Processing in Sensor Networks", Springer Berlin Heidelberg, (2003).

[15] Wand and Dong, et al., "Building Wireless Sensor Networks (WSNs) by Zigbee Technology", Journal of Chongqing university (natural science edition), (2006).

[16] S. Ricardo, J. Sá Silva and F. Boavida, "Evaluating 6lowPAN implementations in WSNs", Proceedings of 9th Conference Computer adores, Portugal, (2009), pp. 1-5.

[17] N. Donggeon, I. Yoon and H. Shin, "Low-Latency Geographic Routing for Asynchronous EnergyHarvesting WSNs", Journal of Networks, vol. 3, no. 1, (2008).

[18] Z. Hao, I. Schizas and G. Giannakis, "Power-efficient dimensionality reduction for distributed channelaware kalman tracking using WSNs", IEEE Transactions on, vol. 57, no.8, (2009), pp. 3193-3207. 
[19] H. Chen, et al., "Agent-based trust model in wireless sensor networks", Proceedings of the Eighth ACIS International Conference on Software Engineering, Artificial Intelligence, Networking and Parallel/Distributed Computing-Volume 03, IEEE Computer Society, (2007).

[20] F. Viani, et al., "Pervasive remote sensing through WSNs: Antennas and Propagation (EUCAP)", 2012 6th European Conference on, IEEE, (2012).

[21] A. Pereira and S. Silva, "Mean square convergence of consensus algorithms in random WSNs", Signal Processing, IEEE Transactions on, vol. 58, no. 5, (2010), pp. 2866-2874.

[22] M. Dohler, et al., "The ARESA project: Facilitating research, development and commercialization of WSNs: Sensor, Mesh and Ad Hoc Communications and Networks", 4th Annual IEEE Communications Society Conference, IEEE, (2007).

[23] N. Guo-Fang, M. Q. Li and J. Li, "Estimation of node localization with a real-coded genetic algorithm in WSNs: Machine Learning and Cybernetics", 2007 International Conference, IEEE, vol. 2, (2007).

[24] K. Raj and S. Goyal, "Perspective of WSNs simulators", (2013), pp. 37-44.

[25] P. Wint Yi, M. Beck and J. Schmitt, "Planning the trajectories of multiple mobile sinks in large-scale, time-sensitive WSNs: Distributed Computing in Sensor Systems and Workshops (DCOSS)", 2011 International Conference, IEEE, (2011).

[26] D. Kleinbaum, et al., "Applied regression analysis and other multivariable methods: Cengage Learning", (2013).

[27] S. Robin, K. Trivedi and A. Puliafito, "Performance and reliability analysis of computer systems: an example-based approach using the SHARPE software package", Springer Publishing Company, Incorporated, (2012).

[28] J. Chang, et al., "The Assessment of Transformer Status Based on Gray Theory Applied Mechanics and Materials", vol. 511, (2014), pp. 1147-1152.

[29] Q. Lulu, E. Winfree and J. Bruck, "Neural network computation with DNA strand displacement cascades", (2011), pp. 368-372.

\section{Author}

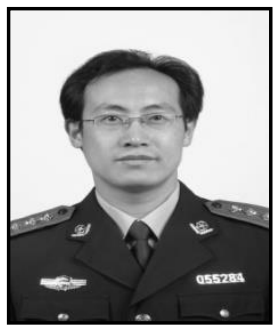

Fawang Han, Male, he was born in December 1972. He is a graduate student and his research directions are computer Network, Internet of Things and Computer basic education etc. 
International Journal of Future Generation Communication and Networking Vol.7, No.5 (2014) 